\title{
A Divide and Conquer matheuristic algorithm for the prize-collecting Steiner tree problem
}

\author{
Murodzhon Akhmedova,b, Ivo Kwee ${ }^{\mathrm{a}, \mathrm{b}}$, Roberto Montemanni ${ }^{\mathrm{a}}$ \\ ${ }^{a}$ Dalle Molle Institute for Artificial Intelligence \\ (IDSIA-USI/SUPSI), Galleria 2, \\ 6928 Manno, Switzerland \\ ${ }^{b}$ Institute of Oncology Research (IOR) \\ Via Vela 6, 6500 Bellinzona, Switzerland
}

\begin{abstract}
The Prize-collecting Steiner Tree Problem (PCSTP) is a well-known problem in graph theory and combinatorial optimization. It has been successfully applied to solve real problems such as fiber-optic and gas distribution networks design. In this work, we concentrate on its application in biology to perform a functional analysis of genes. It is common to analyze large networks in genomics to infer a hidden knowledge. Due to the NP-hard characteristics of the PCSTP, it is computationally costly, if possible, to achieve exact solutions for such huge instances. Therefore, there is a need for fast and efficient matheuristic algorithms to explore and understand the concealed information in huge biological graphs. In this study, we propose a matheuristic method based on clustering algorithm. The main target of the method is to scale up the applicability of the currently available exact methods to large graph instances, without loosing too much on solution quality. The proposed matheuristic method is composed of a preprocessing procedures, a heuristic clustering algorithm and an exact solver for the PCSTP, applied on sub-graphs. We examine the performance of the proposed method on real-world benchmark instances from biology, and compare its results with those of the exact solver alone, without the heuristic clustering. We obtain solutions in shorter execution time and with negligible optimality
\end{abstract}

Email addresses: murodzhon@idsia.ch (Murodzhon Akhmedov), ivo.kwee@ior.iosi.ch (Ivo Kwee), roberto@idsia.ch ( Roberto Montemanni)

Preprint submitted to Computers and Operations Research

December 5, 2015

(C) 2015. This manuscript version is made available under the Elsevier user license http://www.elsevier.com/open-access/userlicense/1.0/ 
gaps. This enables analyzing very large biological networks with the currently available exact solvers.

Keywords: Prize-collecting Steiner Tree Problem (PCSTP); Combinatorial Optimization (CO); Matheuristics (M); Genomics (G)

\section{Introduction}

The Steiner Tree Problem (STP) is a well-know problem in graph theory and there exist different variants of the problem in the literature. The prizecollecting Steiner Tree problem (PCSTP) is a generalization of the STP. Given

5 an undirected graph $G=(V, E)$, where the vertices are labeled with prizes $p_{j} \geq 0$ and the edges are labeled with $\operatorname{costs} c_{e}>0$, the goal is to identify a sub-network $G^{\prime}=\left(V^{\prime}, E^{\prime}\right)$ which is also a tree. The target is to minimize the sum of prizes of the vertices in $V \backslash V^{\prime}$ and to minimize the total cost of the edges in $E^{\prime}$. Thus, the corresponding problem is equivalent to the minimization of the following objective function, also known as Goemans-Williamson minimization problem [16]:

$$
G W\left(G^{\prime}\right)=\min \sum_{e \in E^{\prime}} c_{e}+\sum_{v \notin V^{\prime}} p_{v}
$$

The STP and the PCSTP have a wide range of applications, mainly in the design of utilities such as fiber optic networks and district heating networks. Recently applications in biological networks have appeared [7]. The relation between the PCSTP and genomic networks has been identified and it gathered the attention of researchers in [4]. Vertices and edges in these networks are given a score by using gene expression profiling (GEP) experimental data. GEP [1] measures the activity levels of thousands of genes simultaneously, and therefore it is able to provide a global overview of biological functions at cellular level. 20 Genes have defined procedures to produce messenger RNAs (mRNA) [2] A gene is considered to be active at a time if it produces mRNA, and passive otherwise. Thus, GEP measures the activity of each gene at a given point in time, and this can be related to the concurrent mRNA activities. In a gene 
interaction network, every vertex is a gene and every edge between two vertices represents the genetic interaction between the two corresponding genes. Based on GEP data, each vertex in the network is given a prize, which is the mean value of the differential expression of the corresponding gene [5]. The differential expression is the amount of change in expression level of a gene in two different experimental conditions, for instance, tumor tissue versus control tissue. Also, 30 each edge in the network is given a cost, which corresponds to the pairwise correlation level of the expression values of the corresponding gene pair [5]. After having evaluated all vertices and edges in the network, the PCTSP is then used to detect a relevant sub-network (tree). In biologically terms, the sub-network retrieve by the PCSTP has an important meaning [4, because 35 it corresponds to a portion of the interaction network where many genes are highly correlated in terms of their functions and play an important role in the regulations of biological processes. Thus, the PCTSP can help to detect connected neighborhoods in interaction networks where the genes belong to the same biological pathways [5. Based on these considerations, we develop in this work computational approaches for identifying functions of proteins or genes in cancer genomics relying upon PCSTP solutions. To this end, we construct gene-gene interaction networks by gathering information from genomic data. We use raw microarray data, that are commonly used to quantify gene expressions from different samples of tumor tissues and the corresponding control tissues.

${ }_{45}$ Subsequently, we apply the PCSTP on the generated gene interaction networks to perform functional analysis.

In the application of PCSTP in genomics, it is possible to have very large networks. Nevertheless, PCSTP has NP-hard characteristics. Thus, it is computationally costly to find solutions for large instances in reasonable execution time. Taking this fact into account, one needs an efficient and fast matheuristic algorithms to compute the PCSTP solution for large networks. At the same time, we seek methods able to give high quality solutions for inferring the functional biology. Solution quality and running time speed of the algorithm must be considered simultaneously. In this study, we propose a matheuristic that 
large instances in biological applications. The matheuristic is composed of a preprocessing technique, a heuristic clustering algorithm and an exact solution method for PCSTP, applied on sub-graphs. In this work, we develop a solution strategy to efficiently solve the objective function in Eq. (1). The rest of the literature. In Section 3, we present the proposed matheuristic. The experimental studies of matheuristic are reported in Section 4. Our concluding remarks and future work are delivered in the last section of the paper.

\section{Related Work}

The concept of the PCSTP was first introduced by Bienstock et al [6] and the authors contributed pioneering work by developing 3-approximation algorithm to this research area. Several exact solution methodologies were proposed by Ljubic et al [11] and [10]. In these studies, the authors formulated the problem by employing the mixed integer linear programming and a brand-and-cut algorithm was devised to solve the model. They have tested the performance of their methods on real-world benchmark instances. Besides, there are some very distinct studies in the stream of approximation and heuristic algorithms. Canuto et al 14 introduced a multi-start local search heuristic with perturbations. Goemans and Williamson [8] proposed a primal-dual 2-1/(n-1) approximation algorithm with $\mathrm{O}\left(n^{3} \log n\right)$ running time performance. Another matheuristic approach was presented in Klau et al [18, in which the authors studied an incorporation of integer programming into memetic algorithm. Lucena and Resende 9] proposed a method to obtain a lower bound on the problem using mixed integer linear programming.

Different versions of the PCSTP were analyzed by Johnson et al [16], and for the primal-dual 2-approximation algorithm a well-defined strong pruning rule was developed. Klau et al [19] studied the fractional prize-collecting Steiner tree problem on trees. They maximize the ratio of the vertex profits and the 
edge costs plus a fixed cost. Another version of the PCSTP known as the quota problem was studied in Haouari and Chaouachi [20] and Haouari et al [20. In this problem an additional quota constraint is inserted into the model to force the total prize of the nodes selected in the tree to be above a predetermined threshold $Q$. A further study on a quota problem was presented in Haouari et al 22] by considering penalties - different from vertex prizes - for vertices that are not covered by the output tree. Other related problems were finally discussed in Montemanni et al 23.

In the literature, the PCSTP has been successfully applied to similar biological networks to perform functional analysis in 3, 4, 5. The focus in these studies was to analyze the protein-protein interaction networks. As a result of these studies, the authors have detected unknown and unreported functions for some proteins. Moreover, the authors have justified their computational findings with experimental support from biological experiments. This suggests that the output of PCSTP is very promising and indeed valuable when it is applied to bio-genetical graphs.

Another PCSTP application was proposed in [17, in which a heuristic approach was devised to handle huge biological networks. That implementation targeted large networks with a restricted number of vertices with prizes $p_{j}>0$. In contrast, this study focuses on large biological networks with some different properties. It is common to encounter networks with these properties in genomics [5. Basically, all vertices of the network have positive prizes. Since the generated network is based on genomics, it is highly possible that every gene has non-zero mean value of differential expression. This implies that corresponding vertex has positive prize $p_{j}>0$. Another difference of the targeted networks is the ratio of vertex-prizes to the edge-costs. This ratio usually is not extremely high. The final distinction is that $p_{\max }<<D_{\max }$, where $p_{\max }$ is the maximum prize among all vertices and $D_{\max }$ is the maximum value in all-pairs shortest path matrix. Very similar PCSTP application to networks with same properties was proposed in 30 . The main difference is that a single-commodity flow mixed integer linear programming (MILP) formulation of the PCSTP was considered 
study aims to use the state-of-the-art formulation.

\section{Methodology}

In this section, we present our matheuristic algorithm for PSCTP. The approach is composed of three distinct components: a preprocessing procedure, 120 a heuristic clustering algorithm, and any available exact method to solve the PCSTP separately on each cluster.

It is important to note that any exact state-of-the-art solution approach can be incorporated into the proposed matheuristic. In this study, we use the method proposed in Ljubic et al [10].

125 The main idea of the proposed matheuristic approach is to heuristically divide the giant graph into smaller graphs that can be solved separately, before merging back the results. Solving a single large problem instance of PCSTP is potentially computationally costly compared to solving possibly many smaller problem components of that instance. Therefore, the "divide and conquer" paradigm is adopted while designing the matheuristic.

\subsection{Preprocessing}

The main target of preprocessing is to reduce the size of given network instance by removing the vertices and edges with some specific properties in the graph. The overall preprocessing we adopt after some preliminary experiments 135 is composed of two basic procedures, that are available in the literature [11]. The main reason for choosing these procedures is they are simple and computationally efficient. There are some other more sophisticated procedures in the literature, which require the calculation of all-pairs shortest paths repeatedly [10]. Since this study focuses on large graphs, computing all-pairs shortest paths continuously could be computationally expensive. We repeat these procedures until they fail to reduce the graph size further.

Degree-One Test : Consider a vertex $i$ that has only one edge, to vertex $j$. If $p_{i}<c_{i j}$, then the vertex $i$ is removed together with its edge. If $p_{i}>c_{i j}$, 
then the prize of vertex $j$ becomes $p_{j}=p_{j}+p_{i}-c_{i j}$, and vertex $i$ is removed together with its edge.

Degree-Two Test : Consider a vertex $i$ which has only two edges, to vertices $j$ and $k$ respectively. If there is no edge between vertices $j$ and $k$, an edge is added with cost $c_{j k}=c_{i j}+c_{i k}-p_{i}$. In case there already exists such an edge, then the cost of the edge is set to $\min \left(c_{j k}, c_{i j}+c_{i k}-p_{i}\right)$. Finally, vertex $i$ is removed from the graph together with its edges.

\subsection{A Divide and Conquer Heuristic Clustering}

The proposed heuristic clustering algorithm separates input graph $G$ into independent portions of smaller graphs $G_{s}$ according to minimization objective function (1). At first, the algorithm groups vertices into clusters. Two vertices are clustered together if the shortest path distance between them is smaller than the prize of one of the two vertices. Subsequently, smaller graphs are constructed by considering all vertices belonging to same clusters. The heuristic clustering uses the pre-calculated all-pairs shortest path matrix obtained by Johnson's algorithm [16].

The performance of heuristic clustering is highly dependent on the structure of input graph. The targeted graph instances in this study have particular characteristics, as discussed in section 2 . These make the clustering algorithm practically suitable to be used on them.

The pseudocode of the heuristic clustering approach is provided in Algorithm 165 1. Before starting clustering, the algorithm computes all-pairs shortest path distance matrix $D$ of graph $G$, where $D_{i j}$ is the shortest path distance between the vertices $i$ and $j$. The clustering of vertices is simply based on the comparison of the shortest path distances against prizes. If the shortest path distance between two vertices is smaller than the prize of one of these vertices, then those two vertices are clustered together. More formally, vertices $i$ and $j$ are clustered together if they satisfy the clustering condition, which is $D_{i j}<p_{i}$ or $D_{i j}<p_{j}$. The heuristic starts by arbitrarily selecting a vertex $i$ and permanently labeling it with cluster identifier $I D$. Then, all the other vertices are analyzed one by 
one with respect to vertex $i$, and the vertices satisfying the clustering condition vertex satisfying the clustering condition and set $K$ is empty, so the first iteration of the algorithm is terminated. The heuristic determines the first cluster $G_{1}$ at the end of the first iteration. It is worthy to mention that the set of tem- 

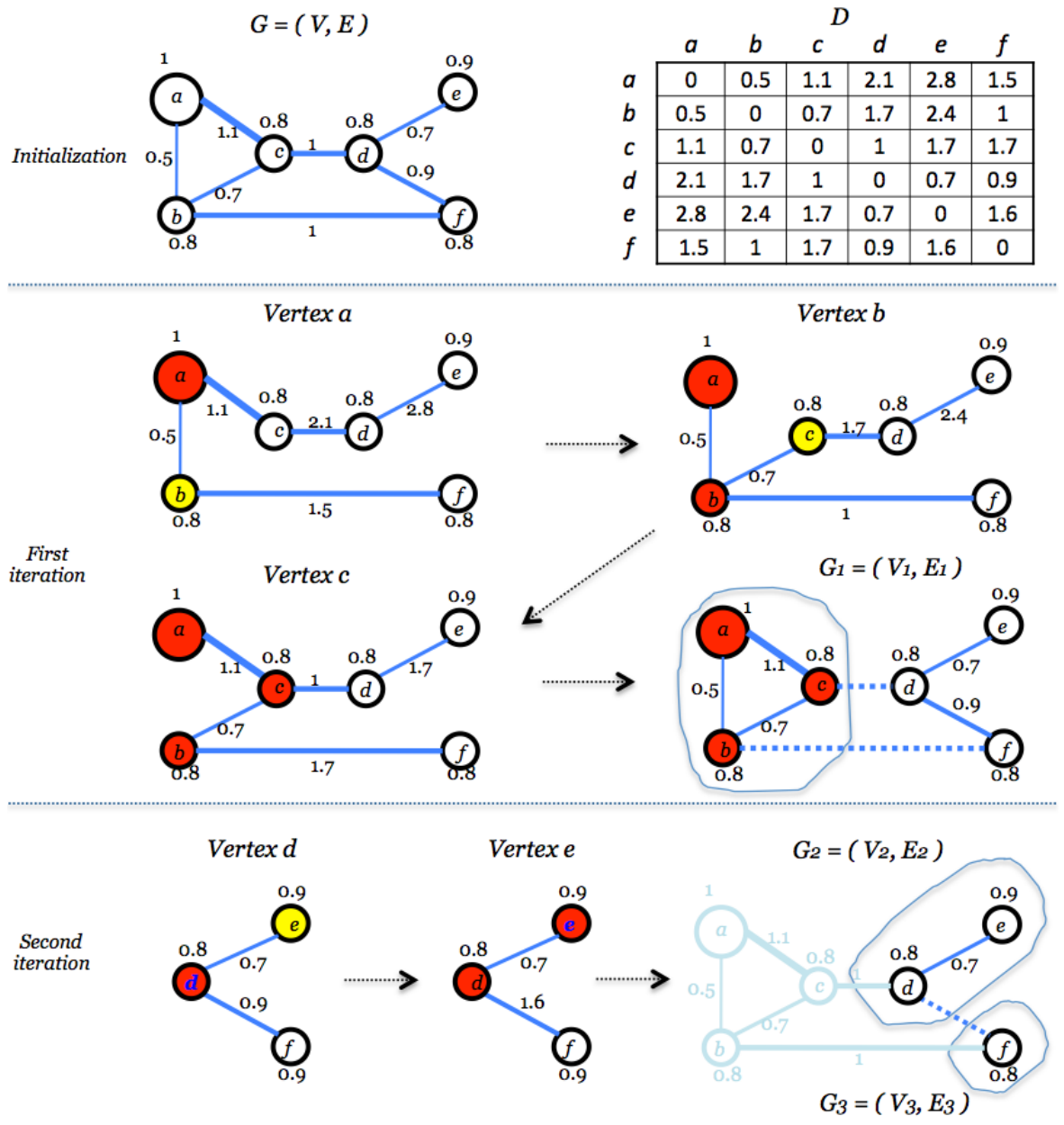

Figure 1: Example of application of the clustering algorithm. Initialization. All-pairs shortest path distance matrix $D$ is calculated for given graph G. First iteration. The shortest path distances from the selected vertex to all the other vertices are represented on the edges. Permanently labeled vertices are colored in red, temporarily labeled vertices are colored in yellow. The heuristic obtains the first cluster $G_{1}$. Second iteration. The same procedure is repeated, and the algorithm obtains clusters $G_{2}$ and $G_{3}$. 


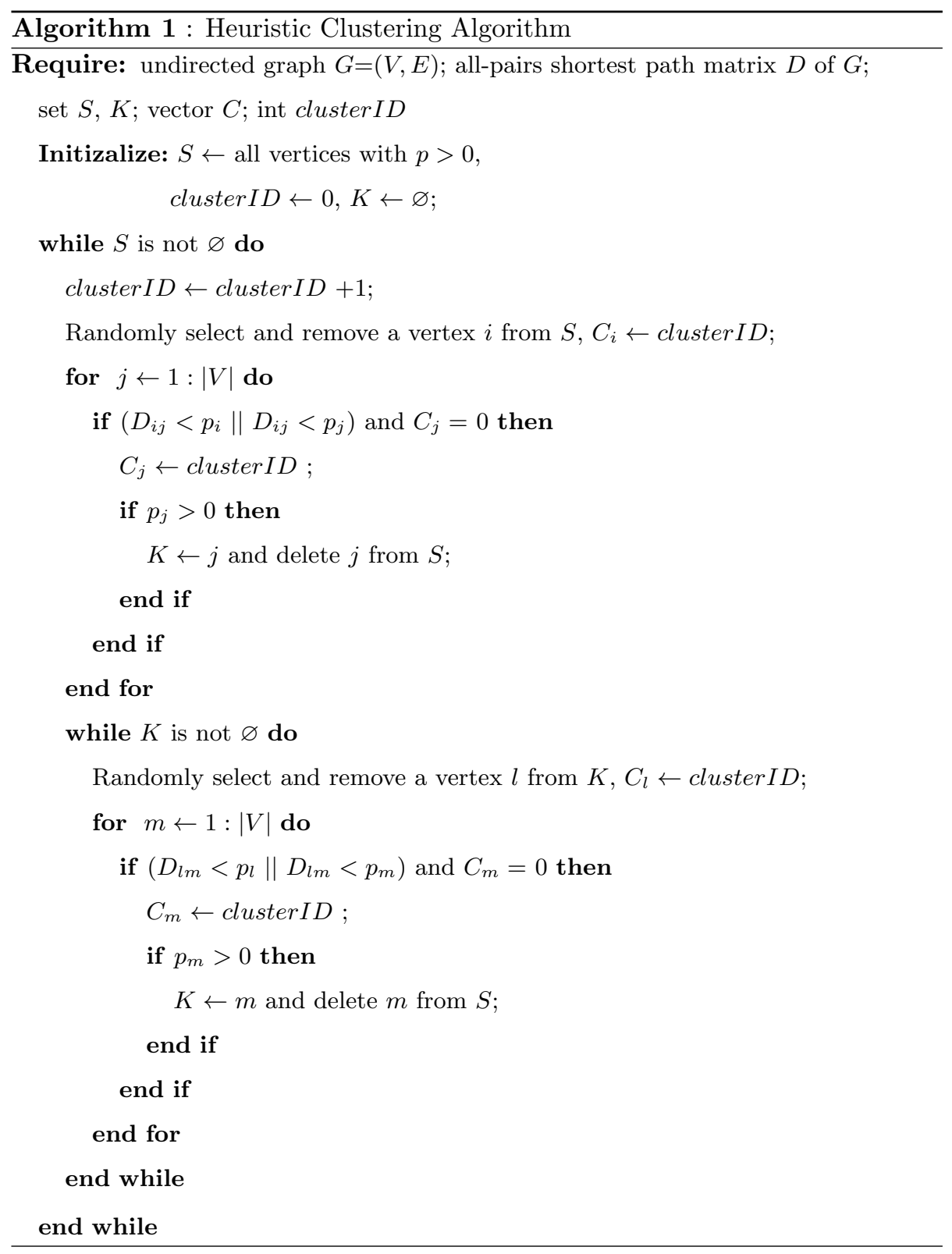


porary labeled vertices $\mathrm{K}$ only contains a single vertex at each step; however, it is not necessarily the case in general. Afterwards, the algorithm disregards the permanently labeled vertices from computation, and continues with the second iteration by arbitrarily selecting vertex $d$ from the rest of the vertices, followed by vertex $e$ later on. The heuristic performs the same procedures and obtains the clusters $G_{2}$ and $G_{3}$ at the end of the second iteration.

The worst-case performance of the heuristic clustering is $O\left(|V|^{4}\right)$ and the proof is straightforward.

\subsection{Solving the PCSTP on the clusters}

In the literature, there exist several MILPs and different techniques to solve the PCSTP to optimality [11, 13. The method proposed by Ljubic et al [10] is the most efficient one available to the best of our knowledge. In this study, we employ this tool to solve the sub-graphs generated by means of the clustering algorithm. The approach of [10] is based on a MILP and branch-and-cut. In the following paragraphs, we provide our MILP formulation that is slightly different 20 from [10]. These differences mainly arise within the constraints of the model due to the properties of the targeted networks in this study.

The PCSTP sub-network $G_{s}=\left(V_{s}, E_{s}, c_{s}, p_{s}\right)$ is transformed into a Steiner arborescence instance $G_{s}^{\prime}=\left(V_{s}^{\prime}, E_{s}^{\prime}, c_{s}^{\prime}\right)$, where $V_{s}^{\prime}$ is formed by combining $V_{s}$ with artificial root vertex $r$. For every undirected edge $(i, j) \in E$, two directed edges $(i, j)$ and $(j, i)$ are added to $E_{s}^{\prime}$ with the $\operatorname{costs} c_{i j}^{\prime}=c_{i j}-p_{j}$ and $c_{j i}^{\prime}=$ $c_{j i}-p_{i}$, respectively, where $c_{i j}$ is equal to $c_{j i}$. In addition, for every vertex in $V$, an edge $(r, j)$ is added to $E_{s}^{\prime}$ from root vertex $r$ with corresponding cost of $-p_{j}$. This transformation is illustrated with an example in Figure 2. The subsequent goal is to find a final tree subgraph $T=\left(V_{t}, E_{t}\right)$ for the transformed Steiner arborescence instance $G_{s}^{\prime}$.

The following model uses decision variables $x_{i j} \in\{0,1\}$ and $y_{i} \in\{0,1\}$ which are defined as follows: 

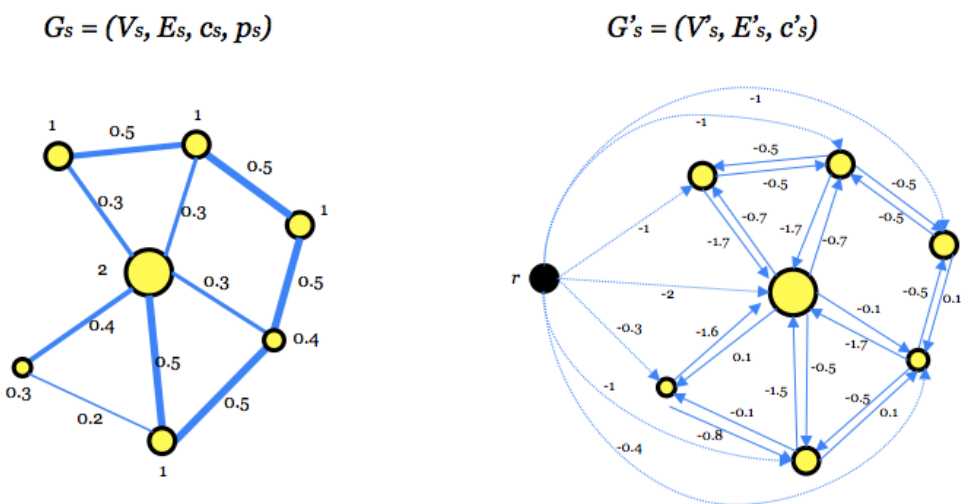

Figure 2: The transformation of the PCSTP instance into the Steiner Arborescence instance.

$$
\begin{aligned}
& { }_{235} \quad x_{i j}= \begin{cases}1 & \text { if }(i, j) \in E_{t}, \quad \forall(i, j) \in E_{s}^{\prime} \\
0 & \text { otherwise }\end{cases} \\
& y_{i}= \begin{cases}1 & \text { if }(i) \in V_{t}, \quad \forall(i) \in V_{s}^{\prime} \\
0 & \text { otherwise }\end{cases}
\end{aligned}
$$

The following notation is also employed: $S \subset V_{s}^{\prime}$ is a set of vertices, and $S^{\prime}=V_{s}^{\prime} / S$ is the complement of that set. The $\delta^{+}(S)=\left\{(i, j) \mid i \in S, j \in S^{\prime}\right\}$ and $\delta^{-}(S)=\left\{(i, j) \mid i \in S^{\prime}, j \in S\right\}$ are two set of directed cuts induced by $S$ and $S^{\prime}$, and $x(A)=\sum_{i j \in A} x_{i j}$.

It should be noted that the PCSTP has a feasible solution only if the root vertex has a single outgoing edge. Accordingly, the mathematical model for the problem defined on the Steiner aborescence is [11:

$$
\begin{aligned}
\text { Minimize } & \sum_{i j \in E_{s}^{\prime}} c_{i j}^{\prime} x_{i j}+\sum_{i \in V_{s}^{\prime}} p_{i} \\
\text { s. t. } & \sum_{j i \in E_{s}^{\prime}} x_{i j}=y_{i} \quad \forall i \in V_{s}^{\prime} \backslash\{r\} \\
& x\left(\delta^{-}(S)\right) \geq y_{i} \quad \forall i \in V_{s}^{\prime}
\end{aligned}
$$




$$
y_{r}=1
$$

$$
\sum_{r i \in E_{s}^{\prime}} x_{r i}=1
$$

$$
x_{r j} \leq 1-y_{i}
$$$$
\forall i<j, i \in V_{s}^{\prime}
$$

$$
x_{i j}+x_{j i} \leq y_{i} \quad \forall i \in V_{s}^{\prime} \backslash r
$$

$$
x_{i j} \in\{0,1\}, y_{i} \in\{0,1\} \quad \forall i \in V_{s}^{\prime} \backslash\{r\},(i, j) \in E_{s}^{\prime}
$$

The objective function (2) minimizes the sum of total edge costs in the solution tree and the constant term. The role of the constant term in the objective function is for obtaining a consistent solution cost with respect to original PCSTP instance. The constraint set (3) enforces every selected vertex to have one directed incoming edge. Each vertex in a solution tree must have a directed path from artificial root vertex and this condition is ensured by the constraint set (4). Constraint (5) maintains the root vertex to be included into final tree and constraint (6) ensures the root to have a single outgoing edge. The following constraints are added in order to strengthen the model further. The constraint set (7) creates a bijection between the solutions of PCSTP and arborescence problem, and it eliminates a huge number of solutions for PCSTP that correspond to same solution for arborescence problem. The constraint set (8) forces the model to select one edge within the pair of directed edges between two vertices. Finally, the constraint set (9) ensures that the decision variables are binary. The interested reader may refer to [10] for further details and explanation about the solution approach. 


\section{Experimental Results}

The matheuristic approach is evaluated on two different problem sets and is compared to the exact method described in [10] (used already as an inner solver by our approach) on those problem sets. We have re-implemented the whole approach discussed in [10. In order to justify the correctness and performance of our re-implementation, we have tested it as a preliminary step.

\subsection{Results of our re-implementation of the exact approach [10]}

We have re-implemented the approach discussed in [10] in $\mathrm{C}++$ and have employed the Boost Graph Library [27] for handling large graphs efficiently. IBM ILOG CPLEX 12.6 28] was used as a MILP solver. The computational studies have been performed on a server equipped with an $\operatorname{Intel}(\mathrm{R}) \operatorname{Xeon}(\mathrm{R})$ CPU E5320 1.86GHz processors and $32 \mathrm{~GB}$ of shared memory. A single core was used for the experiments. On the other hand, the experimental results of 10 had been obtained on a Pentium IV $2.8 \mathrm{GHz}$ computer with $2 \mathrm{~GB}$ of memory, and by employing version 8.1 of ILOG CPLEX.

By employing our re-implementation, we have been able to reproduce the same results for all the instances considered in [10. For illustrative purposes, we report the results for the Cologne I instances in Table 1 , where a comparison with findings reported in [10] is provided. The first column of the table contains instance names. The second and the third columns report the size of the instances. The "DHEA [10 time(s)" column corresponds to the running time of approach reported in [10. The "DHEA time(s)" column contains the running time of our re-implementation of the same approach.

It is difficult to compare the running times of the approaches fairly due to the usage of different machines, solvers and tools in the implementation. The machines are comparable with a little disadvantage for our computer [26]. Taking this fact into account, we can argue that our re-implementation is a little bit advantageous in running time. However, this certainly depend on the upgraded version of CPLEX software and the usage of most recent libraries for graphs. 
Table 1: Results obtained by our re-implementation of the exact approach [10].

\begin{tabular}{c|c|c|c|c} 
Instance & $\mathrm{V}$ & $\mathrm{E}$ & DHEA [10] time(s) & DHEA time(s) \\
\hline i01M1 & 768 & 69077 & 2.9 & 3.0 \\
\hline i01M2 & 768 & 69077 & 487.8 & 167.3 \\
\hline i01M3 & 768 & 69077 & 1195.8 & 694.1 \\
\hline i02M1 & 769 & 69140 & 2.9 & 3.9 \\
\hline i02M2 & 769 & 69140 & 598.2 & 306.5 \\
\hline i02M3 & 769 & 69140 & 1810.9 & 813 \\
\hline i03M1 & 771 & 69100 & 3.1 & 5.0 \\
\hline i03M2 & 771 & 69100 & 326.8 & 110.3 \\
\hline i03M3 & 771 & 69100 & 755.9 & 603.9 \\
\hline i04M1 & 761 & 68907 & 2.8 & 1.3 \\
\hline i04M2 & 761 & 68907 & 22.6 & 9.3 \\
\hline i04M3 & 761 & 68907 & 77.7 & 26.2 \\
\hline i05M1 & 761 & 68934 & 2.8 & 1.0 \\
\hline i05M2 & 761 & 68934 & 122.9 & 60.6 \\
\hline i05M3 & 761 & 68934 & 399.4 & 325 \\
\hline
\end{tabular}

\subsection{Testing the matheuristic approach on real-life biological instances}

The first set of instances used to evaluate the performance of our matheuristic method is composed of real-world biological benchmark instances from the literature. These medium sized protein-protein interaction graphs are taken from Dittrich et al [5] to perform computational analysis. These network instances provide an important information for lyphoma cancer and the detailed description about them can be found in [5].

The second set of instances has been generated by us based on gene expression profiling data of lymphoma cancer patients available in Gene Expression Omnibus repository In particular, this study focuses on Diffuse Large B-Cell Lymphoma (DLBCL) cancer. There exist two DLBCL cancer types which are an activated $B$ cell (ABC) and the germinal center B cell (GCB). The target is to identify signatures that are the group of genes relevant for cancer and have discriminative effects in type classification. In order to achieve this goal,

\footnotetext{
${ }^{1}$ http://www.ncbi.nlm.nih.gov/geo/
} 
Table 2: Preprocessing results of Dittrich et al [5] test instances.

\begin{tabular}{c||c|c|c|c|c} 
Instance & $\mathrm{V}$ & $\mathrm{E}$ & $\mathrm{V}$ & $\mathrm{E}$ & Preprocessing time(s) \\
\hline HCMV & 3863 & 29293 & 3102 & 28331 & 4.62 \\
\hline met_mic1 & 3523 & 4345 & 1788 & 2731 & 1.54 \\
\hline met_mic2 & 3514 & 4332 & 1472 & 2402 & 1.94 \\
\hline met_mic3 & 2853 & 3335 & 881 & 1479 & 1.35 \\
\hline lymphoma & 2034 & 7756 & 1563 & 7285 & 1.75 \\
\hline dros_001 & 5226 & 93394 & 4412 & 92580 & 32.07 \\
\hline dros_005 & 5226 & 93394 & 4412 & 92580 & 31.63 \\
\hline dros_0075 & 5226 & 93394 & 4412 & 92580 & 31.69
\end{tabular}

we have constructed gene-gene interaction graphs, where vertex $j$ represents gene $j$. The node prize is associated with the differential expression value of two cancer types for each gene $p_{j}=\left|E_{A B C}-E_{G C B}\right|$, where $E_{A B C}$ and $E_{G C B}$ are equivalent to the mean of gene expression values of $\mathrm{ABC}$ and $\mathrm{GCB}$ cancer patients for the corresponding gene, respectively. Edge-cost $c_{i j}$ is associated with the correlation value $r_{i j}$ of gene expression values between two genes $i$ and $j$. Each edge is added to the network if the corresponding correlation value is bigger than some threshold value. We have chosen the threshold values as $r_{i j}=\{0.5,0.6,0.7\}$. These instance are bigger than the previous ones in the 325 field, and they consist of graphs with upto 21049 vertices and 293113 edges. It is common to encounter networks with such sizes in genomics. These instances can be made available to other researchers upon request.

We have applied the preprocessing techniques described in Section 3.1 on each test instance before feeding it to the algorithms. Table 2 summarizes the preprocessing results for the test instances discussed in [5]. The first column corresponds to the name of instances. The second and third columns report the original size of input graphs. The fourth and fifth columns show the size of the residual graphs after preprocessing. The last column contains the execution time of the preprocessing procedures.

Table 3 reports the computational results for the instances described in [5]. The first and second columns contain instance names and the objective value 
Table 3: Results of Dittrich et al [5] test instances with and without preprocessing.

\begin{tabular}{c||c||c|c|ccc||c|ccc}
\cline { 3 - 10 } & \multicolumn{9}{c|}{} & \multicolumn{4}{c||}{ With Preprocessing } & \multicolumn{4}{c}{ Without Preprocessing } \\
\hline Instance & OPT & DHEA(s) & MATH(s) & Clusters & OPT-Gap(\%) & DHEA(s) & MATH(s) & Clusters & OPT-Gap(\%) \\
\hline HCMV & 7371.53 & 85.01 & 13.26 & 2429 & 0.000 & 154.94 & 12.18 & 2791 & 0.000 \\
\hline met_mic1 & 11346.93 & 26.42 & 15.12 & 944 & 0.000 & 42.79 & 21.02 & 1749 & 0.000 \\
\hline met_mic2 & 16250.24 & 14.81 & 4.96 & 900 & 0.424 & 22.07 & 3.91 & 1238 & 0.424 \\
\hline met_mic3 & 16919.62 & 3.96 & 1.95 & 420 & 0.022 & 7.78 & 2.05 & 713 & 0.182 \\
\hline lymphoma & 3341.89 & 3.44 & 3.03 & 950 & 0.000 & 2.85 & 2.64 & 1395 & 0.000 \\
\hline dros_001 & 8273.98 & 28136.72 & 7756.16 & 3143 & 0.056 & 30144.22 & 12058.76 & 3940 & 0.056 \\
\hline dros_005 & 8121.31 & 38278.76 & 34838.19 & 999 & 0.000 & 20130.25 & 29386.68 & 1567 & 0.000 \\
\hline dros_0075 & 8039.86 & 12725.58 & 9530.6 & 750 & 0.000 & 8036.81 & 10546.51 & 1277 & 0.000 \\
\hline
\end{tabular}

of each optimal solution. We provide the results obtained by the proposed matheuristic approach both with and without preprocessing. The "DHEA(s)" column contains the running time data of the approach discussed in [10, and it also includes the preprocessing time (if applied) in order to have a fair comparison baseline. The "MATH(s)" column reports the execution time of the matheuristic clustering approach (embedding the exact approach in 10 as an inner solver). The number of clusters obtained by applying the procedure described in Section 3.2 is indicated in the "Clusters" column for each instance.

345 The number of clusters also includes the clusters consisting of just a single vertex. The execution time for the matheuristic includes the preprocessing time (if applied), the time consumed by the heuristic clustering algorithm, and the total time needed to solve all the clusters with the method described in [10]. The "OPT-Gap(\%)" column indicates the optimality gap percentage between the solutions provided by the matheuristic method and the exact approach [10].

The results show that the matheuristic method with preprocessing is able to find solutions in shorter running times than DHEA. On the other hand, optimality gaps are negligible, especially for bioinformatics practitioners. The contribution of the preprocessing procedures to the total execution time data of DHEA is favorable for all the instances except for the last two cases, where it is advantageous not to apply preprocessing for faster running times. For some instances the matheuristic method without preprocessing is faster compared to 
Table 4: Presprocessing results of DLBCL test instances

\begin{tabular}{c||c|c|c|c|c} 
Instance & $\mathrm{V}$ & $\mathrm{E}$ & $\mathrm{V}$ & $\mathrm{E}$ & Preprocessing time(s) \\
\hline GSE4732 & 2407 & 24392 & 1385 & 23370 & 5.67 \\
\hline GSE4475 & 13211 & 81023 & 4753 & 72565 & 162.39 \\
\hline GSE22470 & 13211 & 129103 & 5665 & 121557 & 234.90 \\
\hline GSE10172 & 13211 & 191646 & 13202 & 191637 & 2.99 \\
\hline GSE10846 & 21049 & 293113 & 3576 & 275640 & 1185.53 \\
\hline GSE19246 & 21049 & 126208 & 2567 & 107726 & 532.50 \\
\hline GSE31312 & 21049 & 166598 & 4800 & 150335 & 649.78 \\
\hline GSE23501 & 21049 & 142301 & 3535 & 124787 & 703.94 \\
\hline GSE19246g & 42450 & 1001206 & 8070 & 966826 & 8163.01 \\
\hline GSE10846g & 42450 & 1046485 & 12601 & 1016636 & 8289.28 \\
\hline GSE23501g & 42450 & 1112354 & 14565 & 1084469 & 7617.63 \\
\hline
\end{tabular}

the variant with preprocessing. Preprocessing is therefore not always convenient. Overall optimality gaps are lower than $1 \%$ for all test cases.

Table 4 summarizes the results of the preprocessing procedures on the second set of instances, , while Table 5 reports the complete results, using the same format as in Table 3. For the first 8 instances of Table 5, a maximum computation time of 50000 seconds is imposed. The results show that the matheuristic clustering, both with and without preprocessing, is significantly faster than standard DHEA. For DHEA, contribution of the preprocessing to total running time is significant on these large graphs and without preprocessing it could not solve three instances within the time or memory limits. For the last 3 instances, we set the computational time limit to 100000 seconds since the instances are much larger. DHEA was not able to solve these large instances, either with or without preprocessing, due to time or memory limits. In these cases, apart from GSE23501g (where an upper bound of 12929.78 - anyway worse than that provided by the matheuristic method - was found), DHEA was not able to retrieve a feasible solution, while lower bounds were provided. These lower bounds, together with the cost of the heuristic solutions provided by the matheuristic approach are provided in column "OPT" for these instances. It is however important to observe that the gap between the available lower and 
Table 5: Results of DLBCL test instances with and without preprocessing.

\begin{tabular}{l||c||c|ccc||cc|cc}
\cline { 3 - 11 } & \multicolumn{4}{c}{} & \multicolumn{4}{c||}{ With Preprocessing } & \multicolumn{4}{c}{ Without Preprocessing } \\
\hline Instance & OPT & DHEA(s) & MATH(s) & Clusters & OPT-Gap(\%) & DHEA(s) & MATH(s) & Clusters & OPT-Gap(\%) \\
\hline GSE4732 & 398.86 & 134.80 & 8.18 & 1241 & 0.839 & 253.44 & 6.25 & 2263 & 0.839 \\
\hline GSE4475 & 988.25 & 16344.49 & 218.52 & 4583 & 0.000 & 28855.08 & 231.73 & 13041 & 0.000 \\
\hline GSE22470 & 1006.45 & 42549.55 & 342.52 & 5504 & 0.009 & $* *$ & 330.15 & 13050 & 0.009 \\
\hline GSE10172 & 354.82 & 30280.01 & 541.85 & 12375 & 0.108 & $* *$ & 545.26 & 12384 & 0.108 \\
\hline GSE10846 & 1480.23 & 7021.58 & 1660.88 & 3052 & 0.070 & 18237.53 & 1481.27 & 20507 & 0.070 \\
\hline GSE19246 & 1641.12 & 5801.55 & 2803.58 & 734 & 0.008 & $*$ & 4163.46 & 19216 & 0.008 \\
\hline GSE31312 & 3350.63 & 8312.06 & 2332.31 & 3659 & 0.022 & 24221.80 & 6165.62 & 19464 & 0.022 \\
\hline GSE23501 & 980.08 & 15301.89 & 844.08 & 3250 & 0.038 & 49239.31 & 692.40 & 20695 & 0.038 \\
\hline GSE19246g & {$[9812.04 ; 9815.79]$} & $*$ & 27125 & 5016 & $\leq 0.038$ & $* *$ & 75790 & 40102 & $\leq 0.038$ \\
\hline GSE10846g & {$[8914.83 ; 8946.50]$} & $*$ & 24117 & 9055 & $\leq 0.355$ & $* *$ & 70033 & 39061 & $\leq 0.355$ \\
\hline GSE23501g & {$[12789.95 ; 12817.36]$} & $* *$ & 61612 & 1245 & $\leq 0.214$ & $* *$ & 97106 & 40089 & $\leq 0.214$ \\
\hline ** Insufficient memory & & & & & & & &
\end{tabular}

upper bounds is always below $0.4 \%$ ).

Interestingly, the matheuristic method with and without preprocessing found the same solutions for all test cases. For some instances the matheuristic method without preprocessing is remarkably faster. An intuitive explanation can be the following one: graphs associated with biological networks present the properties described in Section 2, and on those graphs the clustering algorithm (that is significantly effective) tends to mimic the preprocessing itself, which is therefore automatically incorporated into the main algorithm.

Finally, it is worth noting that optimality gaps for the matheuristic algorithm are always below 1\%, in spite of faster execution times. Moreover, the matheuristic method was able to provide solutions for 2 (large) instances, while the exact solver was not.

\section{Conclusions and Future Work}

We have proposed a matheuristic approach for solving the PCSTP based on a clustering algorithm. The main purpose of the method is to scale up the applicability of the currently available exact methods to large instances, without losing too much on solution quality. The proposed approach has been tested on 
real-world instances constructed by combining protein-protein interaction networks with data obtained from micro-array technology. In addition, we have also tested the overall matheuristic approach on networks generated from real lymphoma data obtained again by micro-array technology. The performance of the matheuristic approach has been compared with the state-of-the-art to show the effectiveness of the heuristic clustering phase. On large graphs, the matheuristic method has obtained solutions in shorter execution time with negligible optimality gaps.

In this study, Ljubic et al 10 exact approach has been used as an inner solver for the matheuristic method. For example, a recent study 29] reports a new algorithm, which can bee seen as an updated version of previous approach.

405 As a future work, we are interested in testing such an updated method as an inner solver of the proposed matheuristic method.

\section{Acknowledgment}

The authors would like to thank Martin Luipersbeck and Ivana Ljubic for the useful discussions on their approaches and the area editor together with the reviewers for their valuable suggestions. M. A. is supported by Swiss National Science Foundation through project 205321-147138/1: "Steiner trees for functional analysis in cancer system biology".

\section{References}

[1] A. L. Tarca, R. Romero, and S. Draghici. Analysis of microarray experiments of gene expression profiling. American journal of obstetrics and gynecology, 195(2):373-388, 2006.

[2] M. B. Gerstein, C. Bruce, J. S. Rozowsky, D. Zheng, J. Du, J. O. Korbel, O. Emanuelsson, Z. D. Zhang, S. Weissman, and M. Snyder. What is a gene, post-ENCODE? History and updated definition. Genome Research, 17: 669-681, 2007. 
[3] N. Tuncbag, S. McCallum, S. C. Huang, and E. Fraenkel. SteinerNet: a web server for integrating omic data to discover hidden components of response pathways. Nucleic Acids Research, 40(Web Server issue):W505-9, 2012.

[4] M. B. Becheta, C. Borgsb, A. Braunsteinc, J. Chayesb, A. Dagkessamanskaiad, J. M. Franoisd, and R. Zecchina. Finding undetected protein associations in cell signaling by belief propagation. PNAS, 108:882-887, 2010.

[5] M. T. Dittrich, G. W. Klau, A. Rosenwald, T. Dandekar, and T. Mueller. Identifying functional modules in protein-protein interaction networks: an integrated exact approach. Bioinformatics, 26:223-231, 2008.

6] D. Bienstock, M. X. Goemans, D. Simchi-Levi, and D. Williamson. A note on the prize collecting traveling salesman problem. Mathematical Progamming, 59:413-420, 1993.

[7] N. Tuncbag, A. Braunstein, A. Pagnani, S. C. Huang, J. Chayes, C. Borgs, R. Zecchina, and E. Fraenkel. Simultaneous reconstruction of multiple signaling pathways via the prize-collecting Steiner forest problem. Journal of Computational Biology, 20(2):124-136, 2013.

[8] M. X. Goemans, and D. P. Williamson. The primal-dual method for approximation algorithms and its application to network design problems, D.S. Hochbaum (Ed.), Approximation Algorithms for NP-hard Problems, 144-191,1997.

[9] A. Lucena, and M. G. C. Resende. Strong lower bounds for the prize-collecting Steiner problem in graphs. Discrete Applied Mathematics, 141:277-294, 2004.

[10] I. Ljubic, R. Weiskircher, U. Pferschy, G. W. Klau, P. Mutzel, and M. Fischetti. An algorithmic framework for the exact solution of the prize-collecting Steiner tree problem. Mathematical Progamming, 105(2):427-449, 2006. 
[11] I. Ljubic, R. Weiskircher, U. Pferschy, G. Klau, P. Mutzel, and M. Fischetti. Solving the prize-collecting Steiner tree problem to optimality. Proceedings of ALENEX, Seventh Workshop on Algorithm Engineering and Experiments, $6876,2005$.

[12] I. Ljubic. Exact and Memetic Algorithms for Two Network Design Problems. PhD thesis, Faculty of Computer Science, Vienna University of Technology, Chapter 4:91-151, 2004.

[13] M. Haouari, S. B. Layeb, and H. D. Sherali. Strength of three MIP formulations for the prize collecting Steiner tree problem with a quota constraint. Electronic Notes in Discrete Mathematics, 36:495-5021, 2010.

[14] S. A. Canuto, M. G. C. Resende, and C. C. Ribeiro. Local search with perturbation for the prize- collecting Steiner tree problem in graphs. Networks, $38: 50-58,2001$.

[15] J. E. Beasley. OR-Library: distributing test problems by electronic mail. Journal of the Operational Research Society, 1069-1072, 1990

[16] D. S. Johnson, M. Minkoff, and S. Phillips. The prize collecting Steiner tree problem: theory and practice. Proc. 11th ACM-SIAM Symp. on Discrete Algorithms, 760-769, 2000

[17] M. Akhmedov, I. Kwee and R. Montemanni. A Fast Heuristic for the Prize-collecting Steiner Tree Problem. Lecture Notes in Management Science, 6:207-216, 2014.

[18] G. W. Klau, I. Ljubic, A. Moser, P. Mutzel, P. Neuner, U. Pferschy, G. Raidl, and R. Weiskircher. Combining a memetic algorithm with integer programming to solve the prize-collecting Steiner tree problem. Genetic and Evolutionary Computation GECCO 2004, 3102:1304-1315, 2004.

[19] G. W. Klau, I. Ljubic, P. Mutzel, U. Pferschy, and R. Weiskircher. The fractional prize-collecting Steiner tree problem on trees. Extended Abstract, ESA 2003, 691-702, 2003. 
[20] M. Haouari, and J. Chaouachi. A hybrid Lagrangian genetic algorithm for the prize-collecting Steiner tree problem. Computers and Operations Research, 33:1274-1288, 2006.

[21] M. Haouari, S. Layeb, and H.D. Sherali. The prize collecting Steiner tree problem: Models and Lagrangian dual optimization approaches. Computational Optimization and Applications, 40:13-39, 2008.

[22] M. Haouari, S. Layeb, and H.D. Sherali. Algorithmic expedients for the Prize Collecting Steiner Tree Problem. Discrete Optimization, 7:32-47, 2010.

[23] R. Montemanni, V. Leggieri and C. Triki. Mixed integer formulations for the probabilistic minimum energy broadcast problem in wireless networks. European Journal of Operational Research, 190(2):578-585, 2008.

[24] E. W. Dijkstra. A note on two problems in connexion with graphs. Numerische Mathematik, 1:269-271, 1959.

[25] R. C. Prim. Shortest connection networks and some generalizations. Bell System Technical Journal,1389-1401, 1957.

[26] J. J. Dongarra. Performance of various computers using standard linear equations software. Technical report CS-89-85, University of Manchester, 2014.

[27] J. Siek, L.Q. Lee and A. Lumsdaine. Boost Graph Library, 2000, http://www.boost.org

[28] IBM ILOG CPLEX Optimization Studio, http://www.cplex.com

[29] M. Fischetti, M. Leitner, I. Ljubic, M. Luipersbeck, M. Monaci, M. Resch, D. Salvagnin, and M. Sinnl, 2014 December, Thinning out Steiner trees: a node-based model for uniform edge costs. Mathematical Programming Computations, 2015. Special Issue: 11th DIMACS Implementation Challenge on Steiner Tree Problems, submitted. 
[30] M. Akhmedov, I. Kwee and R. Montemanni. A Matheuristic Algorithm for the Prize-collecting Steiner Tree Problem. The $3^{\text {rd }}$ International Conference on Information and Communication Technology IEEE, 411-415, 2015. 